\title{
Prevalence of Latent Tuberculosis Infection and Associated Risk Factors Among 1557 Nursing Students in a Context of Low Endemicity
}

\author{
Monica Lamberti ${ }^{*}, 1$, Rossella Uccello, ${ }^{1,}$, Maria Grazia Lourdes Monaco ${ }^{1, \S}$, Mariarosaria Muoio, \\ Nicola Sannolo ${ }^{1}$, Paola Arena ${ }^{1}$, Gennaro Mazzarella ${ }^{2}$, Antonio Arnese ${ }^{1}$ and Giuseppe La Cerra ${ }^{2}$ \\ ${ }^{I}$ Department of Experimental Medicine, Section of Hygiene, Occupational Medicine and Forensic Medicine, Second \\ University of Naples, Naples, Italy \\ ${ }^{2}$ Department of Cardio-Thoracic and Respiratory Science, Second University of Naples, Naples, Italy
}

\begin{abstract}
Introduction: The risk of tuberculosis (TBC) in nurses is related to its incidence in the general population. Nursing students involved in clinical training could be exposed to occupational risks similar to those of healthcare workers (HCWs). To better understand the epidemiology of nosocomial TBC among nurses in a context of low endemicity, we recruited a cohort of young nursing trainees at the Second University of Naples.

Methods: A screening programme for LTBI in nursing students was conducted between January 2012 and December 2013, at the Second University of Naples, with clinical evaluations, tuberculin skin test (TST) and, in positive TST student, the interferon-g release assays (IGRA). Putative risk factors for LTBI were assessed by a standardized questionnaire.

Results: 1577 nursing students attending the Second University of Naples have been submitted to screening programme for TBC. 1575 have performed TST as first level test and 2 Quantiferon test (QFT). 19 students were TST positive and continued the diagnostic workup practicing QFT, that was positive in 1 student. Of the 2 subjects that have practiced QFT as first level test only 1 was positive. In 2 students positive to QFT test we formulated the diagnosis of LTBI by clinical and radiographic results.

Conclusion: The prevalence of LTBI among nursing students in our study resulted very low. In countries with a low incidence of TBC, the screening programs of healthcare students can be useful for the early identification and treatment of the sporadic cases of LTBI.
\end{abstract}

Keywords: Health surveillance, nursing students, occupational exposure, quantiferon, tuberculosis, tuberculin skin testing.

\section{INTRODUCTION}

The risk of transmission of Mycobacterium tuberculosis (TB) between patients and health care workers (HCWs) is well recognized even in low-income countries due to abandonment of vaccination campaigns, immigration flows, wide diffusion of primary or secondary immunosuppression, poor efficacy of vaccines currently in use [1,2].

One third of the World's population is estimated to be latently infected with Mycobacterium tuberculosis. People with latent TBC infection (LTBI) do not show symptoms of $\mathrm{TBC}$ and are not infectious, but they are at risk of developing active disease and becoming infectious [3].

The overall incidence of TBC in Italy in last years was $7,85 / 100.000$ with approximately 4.400 new TBC cases annually [4].

\footnotetext{
*Address correspondence to this author at the Department of Experimental Medicine, Section of Hygiene, Occupational Medicine and Forensic Medicine, School of Medicine, Second University of Naples, Naples, Italy; Tel: +39 081566 5901; Fax: +39081566 5898;

E-mail: Monica.LAMBERTI@unina2.it

${ }^{\S}$ Contributed equally to this work.
}

In HCWs, the risk of TBC infection is increased by exposure to patients with infectious disease, insufficient use of protective equipment such as respirators, and working conditions, particularly in inadequately ventilated areas and when conducting techniques which involve exposure to contaminated aerosols $[5,6]$.

Nursing students involved in clinical training could be exposed to occupational risks similar to those of HCWs. In fact, the annual risk of TBC infection (ARTI) among nursing trainees was found to be $5 \%$ in countries with a high incidence of TBC, 3 times higher than the $1.5 \%$ estimated ARTI for the general population [7]. Therefore, the screening for LTBI of both healthcare workers and students is recommended too in low-incidence countries [8], in order to obtain an early diagnosis of cases and prevent progression to active disease. The main purpose of this study was to evaluate the prevalence of LTBI among nursing students, in a context of low endemicity such as the Italian one.

\section{MATERIALS AND METHODS}

The survey was carried out, between January 2012 and December 2013, at the Second University of Naples. All nursing students and paediatric nursing students, attending 
$1^{\text {st }}$ and $3^{\text {rd }}$ year were actively summoned to undergo TBC screening. According to the CDC guidelines our hospital is classified as a "low risk" institution [9].

Information on the following variables was collected using a standardized questionnaire: Age, gender, time of attendance in health care sector, family history of TBC, BCG vaccination, place of birth, prior TST, workplace and chest radiographic findings. BCG vaccination was verified by scars or vaccination records.

Tuberculin Skin Test (TST) was performed by trained personnel following standard procedures. In brief, $0.1 \mathrm{~mL}(2$ TU) of purified protein derivative (PPD, RT23; Statens Serum Institute, Copenhagen, Denmark) is injected. The TST is administered to the volar side of the forearm of the participants and read 72 to 96 hours after the application. A positive TST was defined as an induration measuring $\geq 10$ $\mathrm{mm}$ in healthy subjects [10]. The transverse diameter of the induration is measured by experienced personnel.

All the TST positive cases were also tested with an Interferon-Gamma Release Assay (IGRA; QuantiFERON ${ }^{\circledR}$ TB-Gold Cellestis, Carnegie, Australia) to confirm the diagnosis of LTBI, because of its major specificity compared with conventional TST [11].

The blood samples were treated as recommended by the manufacturer. Briefly, $1 \mathrm{ml}$ of whole blood was sampled in each of the three QFT tubes containing either TB specific antigen (ESAT-6, CFP-10 and TB7.7), no antigen (negative control) or mitogen antigen (positive control) and tubes were incubated at $37^{\circ} \mathrm{C}$ overnight before centrifugation, and IFN- $\gamma$ concentrations (IU/ml) was measured by ELISA following the manufacturer protocol. An IFN- $\gamma \geq 0.35 \mathrm{IU} / \mathrm{ml}$ (TB antigens minus negative control) was considered a positive test [12]. All the IGRA positive cases were carefully examined by an infectious diseases specialist and underwent chest radiography.

In subjects with a history of allergy, secondary immunodeficiency or pregnancy were offered the opportunity to take the Quantiferon test as examination of first level.

In fact, although in literature there is no evidence that adverse reactions by Mantoux test can influence the course of pregnancy [13], in order to enhance the protection of pregnant students, Quantiferon was used as examination of the first level to reduce the risk of allergic type reactions to TST and to avoid subjecting the mother to two diagnostic tests in the case of any TST positivity.

Qualitative variables considered are presented in percentages, and quantitative variables as mean and standard deviation (SD).

Frequency analysis of the following variables was carried out: age, sex, country of origin, time of attendance in health care sector, number of vaccinated TST positivity, positive QFT.

All the activities were performed in compliance with the Declaration of Helsinki and current healthcare standards according to the recommendations of the Italian Ministry of Health [14]. All students included in the survey were informed by a physician about the rationale and aims of the survey and a written informed consent was obtained. According to the Italian legislation concerning the guidelines on observational studies, ethical approval for conducting this survey was unnecessary, and on this basis, cross-sectional studies do not require a formal approval by local Institutional Review Boards [15]. Personal information regarding the subjects included in the study was protected according to Italian law [16].

\section{RESULTS}

1577 nursing students, paediatric nursing students of the Second University of Naples have been submitted to Health Surveillance and included in the study from January $1^{\text {st }} 2012$ to December $31^{\text {st }}$ 2013. The main demographic and epidemiological characteristics of the sample are listed in Table 1.

Table 1. Demographic, epidemiological and clinical characteristics of a cohort of nursing students trained at Second University of Naples in Italy.

\begin{tabular}{|l|c|}
\hline Nursing students included in the study (\%) & 1577 \\
\hline Age of students (SD) & $23.6 \pm 5.4$ \\
\hline $\begin{array}{l}\text { Gender } \\
\text { W/M }\end{array}$ & $1020 / 557$ \\
\hline Overseas students & 10 \\
\hline $\begin{array}{l}\text { Country incidence of TBC infection of students } \\
\text { included in the study } \\
\bullet \quad<10 \text { cases/100000 inhabitants per year } \\
\bullet \quad \geq 10 \text { cases/100000 inhabitants per year }\end{array}$ \\
\hline Working seniority (SD) & 1568 \\
\hline $\begin{array}{l}\text { Vaccinated* } \\
\text { n (\%) }\end{array}$ & $9.4 \pm 0.8$ \\
\hline SD: standard deviation; $*$ vaccination verified by scars or vaccination records. \\
\hline
\end{tabular}

Of the 1577 subjects enrolled, 1575 have practiced TST as first level examination and 2 QFT (Fig. 1). 19 (1.2\%) students that have practiced TST as first level were positive, $1543(98 \%)$ were negative and $13(0.8 \%)$ were absent to reading in the window period from 72 to 96 hours after intradermal injection. Induration by TST ranged between $0 \mathrm{e}$ $30 \mathrm{~mm}$. All subjects with positive TST continued the diagnostic workup practicing Quantiferon with the following results: 1 (5.3\%) was positive and 18 were $(94.7 \%)$, negative.

No significant correlation was observed between the size of TST induration and QFT positivity. Of the 2 subjects that have practiced QFT as I level 1 was positive. The average age of all nursing students was found to be $23.6( \pm 5.4)$.

The working seniority of all students was of $0.43( \pm 0.8)$ years. 16 nursing student, positive to TST test, attending the $3^{\text {rd }}$ year of university. All 2 student positive to QFT attending the $3^{\text {rd }}$ year of university.

It was possible to assess the previous BCG vaccination in 9 subjects included in the study. The group consisted of 6 females and 3 males. Of these, 8 have performed TST test as first level examination. 6 of these were positive to the test. Only 1 vaccinated student performed the QFT as a first level 


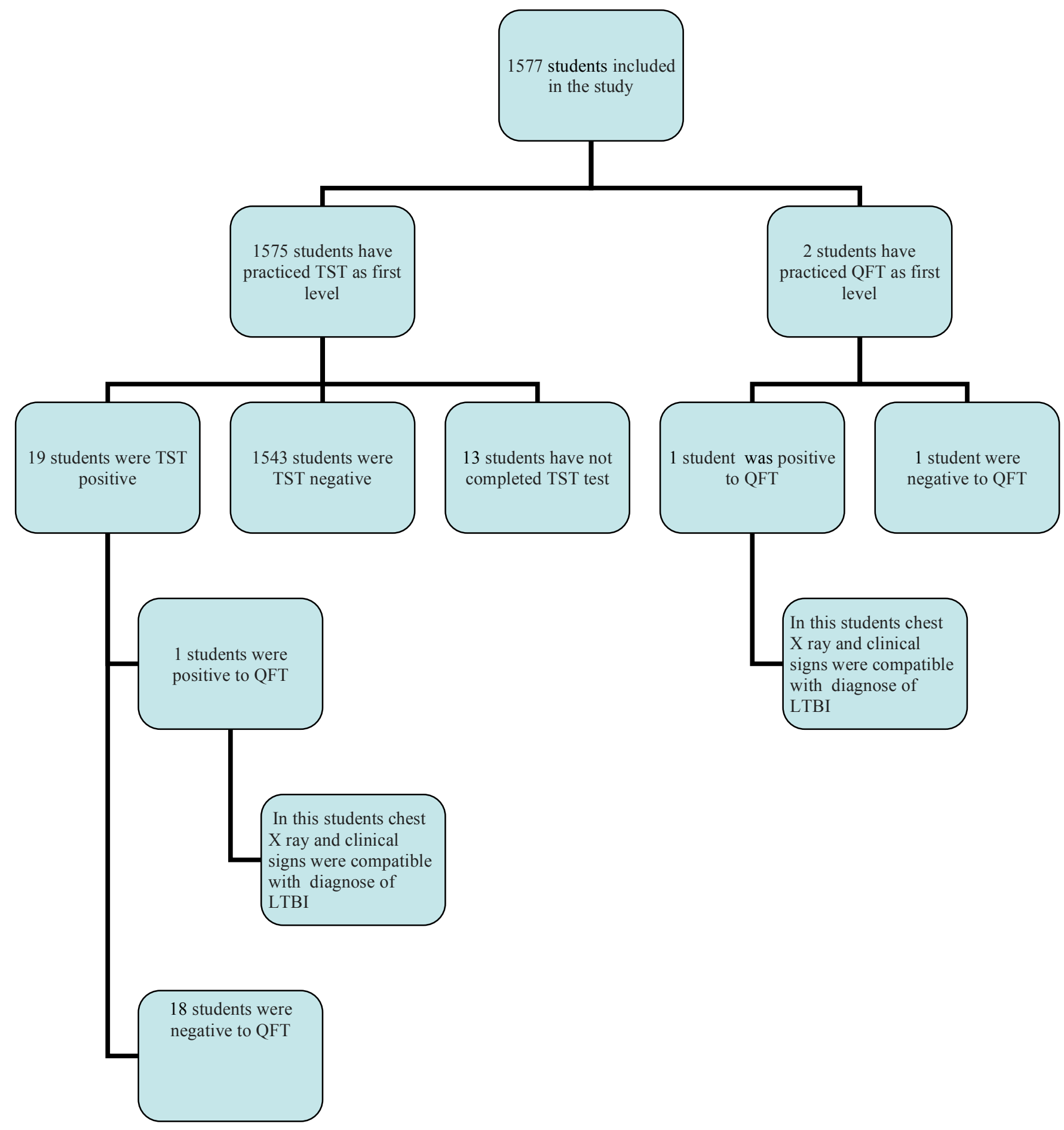

Fig. (1). Study flow chart. TST Tuberculin Skin Test; QFT QuantiFERON ${ }^{\circledR}$ TB-Gold Test; LTBI latent tuberculosis infection.

test and he resulted negative to the test. All subjects with positive TST continued the diagnose practicing Quantiferon with $100 \%$ of negative results.

Among the 1577 students, 10 were overseas students. 9 of these come from a country with a highest incidence of TBC infection higher than the Italian one ( $>10$ cases $/ 100000$ inhabitants per year) [3]. Among the 10 overseas students 7 were negative TST test, and 3 tested positive.

All Italian and overseas students subjects with a positive TST and QFT performed pulmonary consultation and chest
X-ray. Clinical and radiographic signs of active tuberculosis was excluded for all. For this reason, we formulated the diagnosis in 2 students (1 Italians and 1 foreigners) of latent tuberculosis infection (LTBI) with prevalence of $0.12 \%$. Everyone was given an indication to undergo chemoprophylaxis. Only 1 subject adhered to the drug treatment, and follow-up.

Regarding the safety profile of the tuberculin test, none of the subjects who practiced the test showed locoregional or systemic allergic adverse reactions 
Table 2. TST and QFT results in the cohort of nursing students trained at Second University of Naples in Italy.

\begin{tabular}{|c|c|}
\hline $\begin{array}{l}\text { TST as first level examination } \\
\text { - } \text { positive } \\
\text { - } \text { negative } \\
\text { - } \text { absent to reading }\end{array}$ & $\begin{array}{c}1575 \\
19 \\
1543 \\
13\end{array}$ \\
\hline $\begin{array}{l}\text { QFT as first level examination } \\
\text { - } \quad \text { positive } \\
\text { - } \quad \text { negative }\end{array}$ & $\begin{array}{l}2 \\
1 \\
1\end{array}$ \\
\hline $\begin{array}{l}\text { QFT as second level examination in TST positive students } \\
\text { - } \quad \text { positive } \\
\text { - } \quad \text { negative }\end{array}$ & $\begin{array}{c}19 \\
1 \\
18\end{array}$ \\
\hline
\end{tabular}

\section{DISCUSSION}

The growing concern worldwide regarding TBC and nosocomial infection has brought attention to the problem of hospital acquired TB infection among HCWs. However, studies in industrialized countries have shown annual TST conversion rates ranging from $0.1 \%$ to $2 \%$ among unexposed personnel while from $0.5 \%$ to $14 \%$ among highly exposed HCWs [17].

Programs for the screening and treatment of LTBI cases within HCWs are strongly recommended in many countries, with low TBC incidence, including Italy $[8,18]$.

Students and assistants in training are considered potentially exposed to hospital infections because they are assimilated, according to the Italian law, to health professionals (Dgls.no. 81/08) [19].

A systematic review reported that the median estimated annual risk of LTBI among HCWs was $2.9 \%$ in lowincidence countries, against an estimated risk of $7.2 \%$ in countries with a high TB incidence [20].

Our findings showed a prevalence of TST positive cases among all students of only $1.2 \%$. Moreover the diagnosis was confirmed by IGRA testing in only 2 students $(0.12 \%$ incidence). These results are in accordance with the data observed among healthcare students at the beginning of the training period in various occupations (nurses, medical students, physical therapists) by other authors in Europe [21, 22] and are justified by the low risk of contact with infected patients in hospitals for the low prevalence of disease in industrialized countries.

Therefore, based on our data, a biennial risk evaluation of TBC infection, in view of the low incidence of positive TST students, appear to be sufficient to ensure workers health.

The tuberculin skin test in our experience is safe and widely used (no case of loco-regional or systemic allergic reactions) and the use of IGRA in TST positive students reduce the potential occurrence of "false positive" cases due to exposure to atypical mycobacteria or BCG vaccination.

In our study, the influence of age on the risk of tuberculosis infection was evident with a higher percentage of positive cases to TST found in the group of nursing students attending the $3^{\text {rd }}$ year $(84 \%)$.

As shown in literature [23, 24], the prevalence and incidence of tuberculosis in the country of origin can affect the outcome of the screening test. In our sample, the percentage of positive to TST among foreigners was $33 \%$ versus $1 \%$ in the Italian group. All TST positives overseas students come from countries with high TB endemicity (Brazil, Ecuador, Eritrea, Mexico, Moldova, Russia, Ukraine).

In conclusion although we confirm a low prevalence of LTBI among undergraduate healthcare students, in countries with a low incidence of TBC as Italy, LTBI screening programs should include healthcare students before and during clinical training for early identification and treatment of the sporadic cases of LTBI.

\section{CONFLICT OF INTEREST}

The authors confirm that this article content has no conflict of interest.

\section{ACKNOWLEDGEMENTS}

Declared none.

\section{REFERENCES}

[1] Baussano I, Nunn, P, Williams B, Pivetta E, Bugiani M, Scano F. Tuberculosis among health care workers. Emerg Infect Dis 2011; 17: 488-94.

[2] Menzies D, Joshi R, Pai M. Risk of tuberculosis infection and disease associated with work in health care settings. Int $\mathrm{J}$ Tuberc Lung Dis 2007; 11: 593-605.

[3] World Health Organization. WHO Global Tuberculosis Report Geneva: World Health Organization 2013

[4] Ufficio V-Malattie infettive: Direzione generale della prevenzione sanitaria. Ministero della salute Istituto superiore di sanità: La tubercolosi in Italia rapporto 2008 .

[5] Baussano I, Bugiani M, Carosso A, et al. Risk of tuberculin conversion healthcare workers and the adoption of preventive measures. Occup Environ Med 2007; 64: 161-6.

[6] Saleiro S, Santos A, Vidal O, Carvalho T, Torres Costa J, Marques JA. Tuberculosis in hospital department health care workers. Rev Port Pneumol 2007; 13(6): 789-99.

[7] Pai M, Joshi R, Dogra S, et al. Serial testing of health care workers for tuberculosis using interferon-gamma assay. Am J Respir Crit Care Med 2006; 174(3): 349-55.

[8] Mussi TV, Traldi MC, Talarico JN. Knowledge as a factor in vulnerability to tuberculosis among nursing students and professionals. Rev Esc Enferm USP 2012; 46: 696-703.

[9] Guidelines for Preventing the Transmission of Mycobacterium tuberculosis in Health-Care Settings. Department of health and human services. Centers for Disease Control and Prevention 2005.

[10] Duarte R, Amado J, Lucas H, Sapage JM. Treatment of latent tuberculosis infection: Update of guidelines, 2006 Portuguese society of pulmonology. Rev Port Pneumol 2007; 13(3): 397-406.

[11] Trajman A, Steffen RE, Menzies D. Interferon-gamma release assays versus tuberculin skin testing for the diagnosis of latent tuberculosis infection: An overview of the evidence. Pulm Med 2013; 2013: 601737. doi: 10.1155/2013/601737.

[12] Torres Costa J, Silva R, Sá R, Cardoso MJ, Nienhaus A. Results of five-year systematic screening for latent tuberculosis infection in healthcare workers in Portugal. J Occup Med Toxicol 2010; 5: 22. doi: 10.1186/1745-6673-5-22

[13] Centers for Disease Control and Prevention (CDC): Guidelines for Preventing the Transmission of Mycobacterium tuberculosis in Health-Care Settings. MMWR 2005; 54(RR-17): 1-141. http://ww w.cdc.gov/mmwr/preview/mmwrhtml/rr5417a1.htm 
[14] Italian Ministry of Health: Prevenzione della tubercolosi negli operatori sanitari e nei soggetti ad essi equiparati. Roma; 2013. http://www.salute.gov.it/imgs/C_17_pubblicazioni_1901_allegato. pdf

[15] Italian Medicines Agency: Linee guida per la classificazione e conduzione degli studi osservazionali sui farmaci. Gazzetta Ufficiale; 2008. 76, 31/03/2008.http://www.agenziafarmaco.gov.it/ allegati/det_20marzo2008.pdf

[16] Italian Law decree n. 196, 30 June 2003 (article 24). http://www.ca mera.it/parlam/leggi/deleghe/03196dl.htm

[17] Joshi R, Reingold AL, Menzies D, Pai M. Tuberculosis among health-care workers in low- and middle-income countries: A systematic review. PLoS Med 2006; 3(12): e494.

[18] American Thoracic Society/Centers for Disease Control and Prevention/Infectious Diseases Society of America: Controlling tuberculosis in the united states. Am J Respir Crit Care Med 2005, 172: $1169-227$.

[19] Dlgs 9/4/2008, n. 81. Testo unico sulla salute e sicurezza al lavoro. G.U. n.101 30/04/2008 - Supplemento Ordinario n.108
[20] Joshi R, Reingold A, Menzies D, Pai M. Tuberculosis among health-care workers in low- and middle-income countries: A systematic review. PLoS Med 2006; 3: 2376-91.

[21] Schablon A, Beckmann G, Harling M, Diel R, Nienhaus A. Prevalence of latent tuberculosis infection among health care workers in a hospital for pulmonary diseases. J Occup Med Toxicol 2009; 4: 1 .

[22] Durando P, Sotgiu G, Spigno F, et al. Latent tuberculosis infection and associated risk factors among undergraduate healthcare students in Italy: A cross-sectional study. BMC Infect Dis 2013; 13: 443. doi: 10.1186/1471-2334-13-443

[23] Larcher C, Frizzera E, Pretto $\mathrm{P}$, et al: Immunosurveillance for Mycobacterium tuberculosis of health care personnel in a third level care hospital. Med Lav 2012; 103(1): 26-36

[24] Zwerling A, van den Hof S, Scholten J, et al. Interferon-gamma release assays for tuberculosis screening of healthcare workers: A systematic review. Thorax 2012;67(1): 62-70.

(C) Lamberti et al.; Licensee Bentham Open.

This is an open access article licensed under the terms of the Creative Commons Attribution Non-Commercial License (http:/creativecommons.org/licenses/by-nc/3.0/) which permits unrestricted, non-commercial use, distribution and reproduction in any medium, provided the work is properly cited. 